\title{
SOME RESULTS ON THE OPTIMAL SPACING OF MEASUREMENTS IN THE IDENTIFICATION OF STRUCTURAL SYSTEMS*
}

\author{
BY \\ F. E. UDWADIA \\ University of Southern California, Los Angeles
}

\begin{abstract}
This paper deals with finding the optimal measurement locations for a structural system modelled by a single-degree-of-freedom oscillator, so that any one of the parameters to be identified can be estimated with a minimum variance. The measurements are assumed to be taken in a noisy environment, and the paper addresses both linear and nonlinear, nonhysteretic systems. Besides the analytical relations deduced for the optimal measurement locations, it is found that, in general, there may exist measurement locations at which no additional information on the parameter under consideration is generated. For the linear case, the optimal measurement locations are found to be independent of the system response and the actual values of the parameters to be identified. They solely depend on the nature of the excitation used in the identification procedure. Analytical results relating to the optimal measurement locations for minimizing the sum of the variances of the estimates of some of the parameters are also provided.

Introduction. The identification of parameters in dynamic models for building structural systems is a field that is rapidly gaining importance [1-4]. In this paper, we attempt to study the optimal spacing of measurements for a structural system modelled by a single-degree-of-freedom oscillator so that the variance of one of the parameters being identified is minimized.

We start with a linear oscillator and, using Fourier transforms, derive a set a linear algebraic equations. The condition on the measurement frequencies so that the estimated variance (from noisy measurement data) of either the mass parameter, the stiffness parameter or the damping parameter is minimal is derived analytically. It is also found that there may exist a set of frequencies $\omega$, at which no additional information on that parameter is available, yielding no reduction in its estimated variance.

The determination of the optimal measurement frequencies depends solely on the nature of the forcing function used in the identification procedure and is invariant with respect to the actual values of the parameters being estimated.
\end{abstract}

*Received January 10, 1984. 
A numerical example is indicated to illustrate the analytically obtained results. The method is then extended to find the optimal measurement times for structural systems modelled by general nonlinear, second-order differential equations which represent memoryless systems. It is shown, again, that a set of time points may exist at which measurements, if made, will not yield additional information on the parameter of specific concern.

Though the results obtained at this time are purely analytical, it is anticipated that they will help in the design of experiments, especially where data handling and reduction are a major cost concern.

Problem statement. Consider a structure modelled by a single-degree-of-freedom system oscillator subjected to an excitation force $q(t)$. If $x$ is the displacement of such an oscillator, then its equation of motion is

$$
m \ddot{x}+c \dot{x}+k x=q(t), \quad x(0)=0, \quad \dot{x}(0)=0,
$$

where the parameters $m, c$ and $k$ denote the mass, damping and stiffness respectivelyand are assumed to be real numbers. Taking Fourier transforms, this yields

$$
-m \omega^{2} X(\omega)+i c \omega X(\omega)+k X(\omega)=Q(\omega),
$$

so that

$$
1 / X(\omega)=\left(-m \omega^{2}+i c \omega+k\right) / Q(\omega), \quad \omega \in I_{\Omega},
$$

where we shall assume that the division on both sides of equation (2) is possible i.e., there exists an open interval, $I_{\Omega}$, such that for all $\omega \in I_{\Omega}, X(\omega)$ and $Q(\omega)$ are not identically zero.

We shall assume that the parameters $m, k$ and $c$ need to be identified, and we shall direct our interest to finding if there exists a set of frequencies $\omega_{i}, i=1,2, \ldots$ such that the variance of any one of the desired parameters (i.e., $m$, or $k$ or $c$ ) can be minimized by using the data i.e., $X(\omega)$ and $Q(\omega)$ at those specific frequencies.

Relation (3) can be rewritten, after separating the real and imaginary parts, as

$$
\left[\begin{array}{l}
U(\omega) \\
V(\omega)
\end{array}\right]=\left[\begin{array}{ccc}
-\omega^{2} f(\omega) & -\omega g(\omega) & f(\omega) \\
-\omega^{2} g(\omega) & \omega f(\omega) & g(\omega)
\end{array}\right]\left[\begin{array}{c}
m \\
c \\
k
\end{array}\right]+\left[\begin{array}{c}
\varepsilon_{U}(\omega) \\
\varphi_{V}(\omega)
\end{array}\right],
$$

where we have represented

$$
\left.\begin{array}{l}
1 / X(\omega)=U(\omega)+i V(\omega), \\
1 / Q(\omega)=f(\omega)+i g(\omega),
\end{array}\right\}
$$

and the measurements $U(\omega)$ and $V(\omega)$ are corrupted by measurement noise $\varepsilon_{U}(\omega)$ and $\varepsilon_{V}(\omega)$. Defining

$$
\left[a_{j}(\omega)\right] \stackrel{\Delta}{=} \mathbf{a}(\omega) \stackrel{\Delta}{=}\left[-\omega^{2} f(\omega)-\omega g(\omega) f(\omega)\right]^{\mathrm{T}}
$$

and

$$
\left[b_{j}(\omega)\right] \triangleq \mathbf{b}(\omega) \triangleq\left[-\omega^{2} g(\omega) \omega f(\omega) g(\omega)\right]^{\mathrm{T}}
$$


for each value of $\omega=\omega_{i}, i=1,2, \ldots, N$, equation (4) can be written and the BLUE estimator obtained. This can be expressed by the relation

$$
\mathbf{z}=H \theta+\varepsilon,
$$

where

$$
\begin{aligned}
\mathbf{z} & =\left[U\left(\omega_{1}\right) V\left(\omega_{1}\right) U\left(\omega_{2}\right) V\left(\omega_{2}\right) \cdots U\left(\omega_{n}\right) V\left(\omega_{n}\right)\right]^{\mathrm{T}}, \\
H & =\left[\mathbf{a}\left(\omega_{1}\right) \mathbf{b}\left(\omega_{1}\right) \mathbf{a}\left(\omega_{2}\right) \mathbf{b}\left(\omega_{2}\right) \cdots \mathbf{a}\left(\omega_{n}\right) \mathbf{b}\left(\omega_{n}\right)\right]^{\mathrm{T}}, \\
\boldsymbol{\varepsilon} & =\left[\varepsilon_{U}\left(\omega_{1}\right) \varepsilon_{V}\left(\omega_{2}\right) \varepsilon_{V}\left(\omega_{2}\right) \cdots \varepsilon_{U}\left(\omega_{n}\right) \varepsilon_{V}\left(\omega_{n}\right)\right]^{\mathrm{T}} .
\end{aligned}
$$

Assume that the error vector $\varepsilon$ has the statistic

$$
E[\varepsilon]=0 \quad \text { and } \quad R \triangleq E\left(\varepsilon \varepsilon^{\mathrm{T}}\right)=\operatorname{diag}\left(\sigma_{1}^{2}, \sigma_{1}^{2}, \sigma_{2}^{2}, \ldots \sigma_{n}^{2}, \sigma_{n}^{2}\right),
$$

where $\sigma_{i}=\sigma\left(\omega_{i}\right), i=1,2, \ldots, n$. The covariance of the BLUE estimate of the vector $\theta$ becomes [5]

$$
P=\left(H^{\mathrm{T}} R^{-1} H\right)^{-1} \text {. }
$$

where $P$ is a $3 \times 3$ matrix whose diagonal elements $P_{11}, P_{22}$ and $P_{33}$ are the variances in the estimate of $m, c$ and $k$ respectively.

Optimal choice of frequencies, $\omega_{k}$. We shall now attempt to choose the frequencies $\omega_{k} \in I_{\Omega}$ in such a way that the $i$ th element of $P, P_{i i}$, is minimized. To that end we first differentiate relation (10) with respect to $\omega_{k}$ to yield

$$
\frac{\partial P}{\partial \omega_{k}}=-P\left[\frac{\partial H^{\mathrm{T}}}{\partial \omega_{k}} R^{-1} H+H^{\mathrm{T}} R^{-1} \frac{\partial H}{\partial \omega_{k}}-H^{\mathrm{T}} R^{-1} \frac{\partial R}{\partial \omega_{k}} R^{-1} H\right] P,
$$

so that

$$
\left(\frac{\partial P}{\partial \omega_{k}}\right)_{r r}=-2\left[P \frac{\partial H^{\mathrm{T}}}{\partial \omega_{k}} R^{-1} H P\right]_{r r}+\left[P H^{\mathrm{T}} R^{-1} \frac{\partial R}{\partial \omega_{k}} R^{-1} H P\right]_{r r}
$$

Assuming that $\mathbf{a}(\omega), \mathbf{b}(\omega)$ and $\sigma(\omega)$ are continuous functions in $I_{\Omega}$,

$$
\left(\partial H^{\mathrm{T}} / \partial \omega_{k}\right)=\left[0 \ldots 0 \dot{\mathbf{a}}\left(\omega_{k}\right) \dot{\mathbf{b}}\left(\omega_{k}\right) 0 \ldots 0\right] \text {, }
$$

so that

$$
\begin{aligned}
\frac{\partial P_{r r}}{\partial \omega_{k}}= & -\frac{2}{\sigma_{k}^{2}}\left\{P\left[\dot{\mathbf{a}}\left(\omega_{k}\right) \mathbf{a}^{\mathrm{T}}\left(\omega_{k}\right)+\dot{\mathbf{b}}\left(\omega_{k}\right) \mathbf{b}^{\mathrm{T}}\left(\omega_{k}\right)\right] P\right\}_{r r} \\
& +\frac{2 \dot{\boldsymbol{\sigma}}\left(\omega_{k}\right)}{\sigma^{3}\left(\omega_{k}\right)}\left\{P\left[\mathbf{a}\left(\omega_{k}\right) \mathbf{a}^{\mathrm{T}}\left(\omega_{k}\right)+\mathbf{b}\left(\omega_{k}\right) \mathbf{b}^{\mathrm{T}}\left(\omega_{k}\right)\right] P\right\}_{r r} .
\end{aligned}
$$

On expanding, equation (13a) becomes

$$
\begin{aligned}
\frac{\sigma_{k}^{2}}{2} \frac{\partial P_{r r}}{\partial \omega_{k}}= & -\sum_{j, s}\left\{P_{r j} \dot{a}_{j}\left(\omega_{k}\right) a_{s}\left(\omega_{k}\right) P_{s r}+P_{r j} \dot{b}_{j}\left(\omega_{k}\right) b_{s}\left(\omega_{k}\right) P_{s r}\right\} \\
& +\frac{\dot{\sigma}\left(\omega_{k}\right)}{\sigma\left(\omega_{k}\right)}\left\{\left(\sum_{j} P_{r j} a_{j}\right)^{2}+\left(\sum_{j} P_{r j} b_{j}\right)^{2}\right\} .
\end{aligned}
$$


The condition that $P_{r r}$ be extremal then yields

$$
\begin{gathered}
{\left[\sum_{j} P_{r j} \dot{a}_{j}\left(\omega_{k}\right)\right]\left[\sum_{s} P_{s r} a_{s}\left(\omega_{k}\right)\right]+\left[\sum_{j} P_{r j} \dot{b}_{j}\left(\omega_{k}\right)\right]\left[\sum_{s} P_{s r} b_{s}\left(\omega_{k}\right)\right]} \\
-\frac{\dot{\boldsymbol{\sigma}}\left(\omega_{k}\right)}{\boldsymbol{\sigma}\left(\omega_{k}\right)}\left[\left(\sum_{j} P_{r j} a_{j}\right)^{2}+\left(\sum_{j} P_{r j} b_{j}\right)^{2}\right]=0 .
\end{gathered}
$$

LEMMA 1. If a real $\omega_{k} \in I_{\Omega}$ exists such that $f\left(\omega_{k}\right)$ and $g\left(\omega_{k}\right)$ are not zero, and for any $r \in[1,2,3]$,

$$
\sum_{s} P_{s r} a_{s}\left(\omega_{k}\right)=0
$$

then,

$$
\sum_{s} P_{s r} b_{s}\left(\omega_{k}\right)=0 \text { for that value of } r
$$

and vice versa.

Proof. Let us say that we have a sequence of $\omega$ 's, $\omega_{1}, \omega_{2}, \ldots, \omega_{k}, \ldots, \omega_{N}$, where for $\omega_{k}$ relation (15) is valid.

Using relations (8) and (10) we have,

$$
P^{-1}=\sum_{s=1}^{N}\left[\frac{\mathbf{a}\left(\omega_{s}\right) \mathbf{a}^{\mathrm{T}}\left(\omega_{s}\right)+\mathbf{b}\left(\omega_{s}\right) \mathbf{b}^{\mathrm{T}}\left(\omega_{s}\right)}{\sigma_{s}^{2}}\right]
$$

so that

$$
P^{-1}=\left[\begin{array}{ccc}
\sum_{s=1}^{N} \frac{\omega_{s}^{4} \alpha^{2}\left(\omega_{s}\right)}{\sigma_{s}^{2}} & 0 & -\sum_{s=1}^{N} \frac{\omega_{s}^{2} \alpha^{2}\left(\omega_{s}\right)}{\sigma_{s}^{2}} \\
0 & \sum_{s=1}^{N} \frac{\omega_{s}^{2} \alpha^{2}\left(\omega_{s}\right)}{\omega_{s}^{2}} & 0 \\
-\sum_{s=1}^{N} \frac{\omega_{s}^{2} \alpha^{2}\left(\omega_{s}\right)}{\sigma_{s}^{2}} & 0 & \sum_{s=1}^{N} \frac{\alpha^{2}\left(\omega_{s}\right)}{\sigma_{s}^{2}}
\end{array}\right]
$$

where

$$
\alpha^{2}\left(\omega_{s}\right)=f^{2}\left(\omega_{s}\right)+g^{2}\left(\omega_{s}\right)
$$

The determinant $\Delta$ of $P^{-1}$ then becomes

$$
\Delta=\sum \omega_{s}^{2} \alpha^{2}\left(\omega_{s}\right) \cdot\left\{\sum_{s=1}^{N} \frac{\alpha^{2}\left(\omega_{s}\right)}{\sigma_{s}^{2}} \sum_{s=1}^{N} \frac{\omega_{s}^{4} \alpha^{2}\left(\omega_{s}\right)}{\sigma_{s}^{2}}-\left[\sum \frac{\omega_{s}^{2} \alpha^{2}\left(\omega_{s}\right)}{\sigma_{s}^{2}}\right]^{2}\right\},
$$

which by the Cauchy-Schwartz inequality is always $>0$, as it should be, since $P$ is a covariance matrix. Thus the matrix $P$ now becomes

$$
P=\left[\begin{array}{ccc}
A B & 0 & B^{2} \\
0 & C A-B^{2} & 0 \\
B^{2} & 0 & B C
\end{array}\right] / \Delta,
$$


where

$$
\left.\begin{array}{rl}
A=\sum_{s=1}^{N} \frac{\alpha^{2}\left(\omega_{s}\right)}{\sigma_{s}^{2}}, & B=\sum_{s=1}^{N} \frac{\omega_{s}^{2} \alpha^{2}\left(\omega_{s}\right)}{\sigma_{s}^{2}}, \\
C=\sum_{s=1}^{N} \frac{\omega_{s}^{4} \alpha^{2}\left(\omega_{s}\right)}{\sigma_{s}^{2}}, \\
\Delta=B\left(A C-B^{2}\right) .
\end{array}\right\}
$$

Noting relation (6), condition (15) becomes

$$
\begin{aligned}
f\left(\omega_{k}\right)\left[-\omega_{k}^{2} A+B\right] & =0, & & r=1, \\
-g\left(\omega_{k}\right)\left[\omega_{k}\left(C A-B^{2}\right)\right] & =0, & r & =2, \\
f\left(\omega_{k}\right)\left[-\omega_{k}^{2} B+C\right] & =0, & r & =3 .
\end{aligned}
$$

Relation (16) yields

$$
\begin{aligned}
g\left(\omega_{k}\right)\left[-\omega_{k}^{2} A+B\right] & =0, & r & =1, \\
f\left(\omega_{k}\right)\left[\omega_{k}\left(C A-B^{2}\right)\right] & =0, & r & =2, \\
g\left(\omega_{k}\right)\left[-\omega_{k}^{2} B+C\right] & =0, & r & =3 .
\end{aligned}
$$

If $g\left(\omega_{k}\right)$ and $f\left(\omega_{k}\right) \neq 0$ then the two sets of equations become identical, and the result follows.

LEMMA 2.

$$
\frac{\sum P_{r j} b_{j}}{\sum P_{r j} a_{j}}=\frac{g\left(\omega_{k}\right)}{f\left(\omega_{k}\right)}, \quad \sum P_{r j} a_{j} \neq 0, r=1,3
$$

and

$$
\frac{\sum P_{r j} b_{j}}{\sum P_{r j} a_{j}}=-\frac{f\left(\omega_{k}\right)}{g\left(\omega_{k}\right)}, \quad \sum P_{r j} a_{j} \neq 0, r=2 .
$$

Proof. The proof follows from equations (22a) and (22b).

THEOREM 1. For a given forcing function $Q(\omega)$ and any $r \in\{1,2,3\}$, there may exist frequencies $\omega_{k}$ such that the inclusion of data at those frequencies does not yield any improvement in the variance $P_{r r}$ of our estimate of parameter $r$. Specifically, when $\omega_{k} \in I_{\Omega}$ satisfies equation (15), $\omega=\omega_{k}$ is such a frequency.

Proof. Let us imagine that the measurements at the frequencies $\omega_{1}, \omega_{2}, \ldots, \omega_{k-1}$ have been made and that with each measurement, the covariance matrix $P$ is updated. After making the $k$ th measurement at $\omega=\omega_{k}$, the updated covariance matrix becomes [5]

$$
P^{+}=P^{-}-P^{-} H_{k}^{\mathrm{T}}\left[R_{k}+H_{k} P^{-} H_{k}^{\mathrm{T}}\right]^{-1} H_{k} P^{-},
$$

where

$P^{-}$denotes the covariance before the measurement at $\omega_{k}$,

$P^{+}$denotes the covariance after the measurement at $\omega_{k}$,

$H_{k}$ denotes $[\mathbf{a}, \mathbf{b}]^{\mathrm{T}}$ evaluated at $\omega_{k}$, and

$R_{k}=\sigma_{k}^{2} \operatorname{diag}(1,1)$. 
Relation (25) can be rewritten using the notation

$$
L \triangleq P^{-} H_{k}^{\mathrm{T}}
$$

as

$$
P^{+}=P^{-}-L\left[R_{k}+H_{k} P^{-} H_{k}^{\mathrm{T}}\right]^{-1} L^{\mathrm{T}} .
$$

Using relations (6) and (8), we have

$$
L=\left[\begin{array}{cc}
\sum P_{1 j}^{-} a_{j} & \sum P_{1 j}^{-} b_{j} \\
\sum P_{2 j}^{-} a_{j} & \sum P_{2 j}^{-} b_{j} \\
\sum P_{3 j}^{-} a_{j} & \sum P_{3 j}^{-} b_{j}
\end{array}\right]
$$

If relation (15) is valid for some $r$, then

$$
\sum P_{r j}^{-} a_{j}\left(\omega_{k}\right)=\sum P_{r j}^{-} b_{j}\left(\omega_{k}\right)=0,
$$

and by relation (28)

$$
L_{r j}=0, \quad j=1,2 \text {, for that } r .
$$

Consequently, from equation (27) we find

$$
P_{r j}^{+}=P_{r j}^{-}, \quad j=1,2,3 .
$$

We note in passing, using relations (29) and (31),

$$
\sum P_{r j}^{+} a_{j}=\sum P_{r j}^{+} b_{j}=0 \text {. }
$$

COROllaRy 1. For values of $\omega_{k}$ for which $\sum P_{r j} a_{j}\left(\omega_{k}\right)=0$ (for $r=1,3$ ), the variance of the estimate of the $r$ th variable as well as its covariance are unaffected by the measurement $\omega=\omega_{k}$.

Proof. The result follows directly from relation (31).

We note that the frequencies $\omega_{k}$, which do not contain any further information (for any given $r$ ), do not depend on the parameter values $m, c$ and $k$. They are only governed by the nature of the forcing function $Q(\omega)$ and can be calculated before even the measurements are made. They also do not depend on the measured responses.

Corollary 2a. If $g\left(\omega_{k}\right) \neq 0$ and $f\left(\omega_{k}\right) \neq 0$, there exists no $\omega_{k} \in I_{\Omega}$ except possibly $\omega_{k}=0$ for which

$$
\sum P_{2 j} a_{j}\left(\omega_{k}\right)=0
$$

provided the covariance matrix is nonsingular.

Proof. Referring to equations (22) for $r=2$ and noting that $A C-B^{2} \neq 0$ because the determinant of the covariance matrix is nonzero, the equations can only be satisfied by $\omega_{k}=0$.

Corollary 2b. If $g\left(\omega_{k}\right) \neq 0$ and $f\left(\omega_{k}\right) \neq 0$, there always exists an $\omega_{k}, \omega_{k} \in(0, \infty)$, for which $\sum P_{r j} a_{j}\left(\omega_{k}\right)=0, r=1,3$.

Proof. We shall show the case for $r=1$. Using relation $(22 \mathrm{a}), \omega_{k}^{2}=B / A$. From equation (21), $B>0$ and $A>0$. Thus $\omega_{k}=(B / A)^{1 / 2}$.

The proof for $r=3$ follows along similar lines. 
THEOREM 2. The optimal locations for the measurements $\omega=\omega_{k}, \omega_{k} \in I_{\Omega}$, if they exist at all, which minimize the variance in the estimates $P_{r r}$, satisfy the following relations:

$$
\sum P_{r j} \dot{a}_{j}\left(\omega_{k}\right) \frac{g\left(\omega_{k}\right)}{f\left(\omega_{k}\right)} \sum P_{s r} \dot{b}_{s}\left(\omega_{k}\right)-\frac{\dot{\sigma}\left(\omega_{k}\right)}{\sigma\left(\omega_{k}\right)}\left[1+\left(\frac{g\left(\omega_{k}\right)}{f\left(\omega_{k}\right)}\right)^{2}\right] \sum P_{r j} a_{j}=0, \quad r=1,3
$$

when $f\left(\omega_{k}\right) \neq 0$. When $f\left(\omega_{k}\right)=0$, they satisfy the relation

$$
\sum_{s} P_{s r} \dot{b}_{s}\left(\omega_{k}\right)-\frac{\dot{\sigma}\left(\omega_{k}\right)}{\sigma\left(\omega_{k}\right)} \sum_{s} P_{s r} b_{s}\left(\omega_{k}\right)=0, \quad r=1,3
$$

Proof. Using equation (14) and Lemma 2, the result follows. A similar result can be written for the cases when $g\left(\omega_{k}\right) \neq 0$ and $g\left(\omega_{k}\right)=0$ respectively.

Equations (34a) and (34b) express the criteria for finding observation points $\omega_{k}$ such that the mass $m$ or the stiffness $k$ can be optimally identified. We note that the optimal location $\omega_{k}$ of the $k$ th observation point depends in general upon the location of all the previous observation points as contained in $P_{r j}$ and $P_{s r}$.

THEOREM 3. The optimal locations for the measurements $\omega=\omega_{k}, \omega_{k} \in I_{\Omega}$, if they exist at all, which minimize the variance of the damping parameter $c$, satisfy the relation

$$
\left[1-\frac{\omega_{k} \dot{\sigma}\left(\omega_{k}\right)}{\sigma\left(\omega_{k}\right)}\right]\left[g^{2}\left(\omega_{k}\right)+f^{2}\left(\omega_{k}\right)\right]=-\left.\frac{\omega_{k}}{2} \frac{d}{d \omega}\left[g^{2}(\omega)+f^{2}(\omega)\right]\right|_{\omega_{k}} .
$$

Proof. Noting relation (14) and Lemma 2, the result follows. We assume that the covariance matrix is strictly positive definite.

Theorem 3 states that to optimally locate the $k$ th measurement, relation (35) needs to be satisfied. We note that in this case the optimal location of the $k$ th measurement does not depend on the locations of the preceeding measurements and is purely controlled by the nature of the graphs of $f(\omega)$ and $g(\omega)$.

Next let us consider the problem of minimizing the sum of the variances of $l$ out of the 3 parameters. Let $s_{i}, i=1, \ldots, l$ be these $l$ parameters. Let $\Lambda$ be the zero matrix whose $n$th diagonal element is unity if $n=s_{i}, i=1, \ldots, l, s_{i} \in(1,2,3)$. We then have the following result.

THEOREM 4. The frequencies $\omega_{k} \in I_{\Omega}$ which make

$$
\sum_{i=1}^{l} P_{s_{i} s_{i}}, \quad l \leqslant 3
$$

extremal are given by the relation

$$
\mathbf{a}^{\mathrm{T}}\left(\omega_{k}\right) P \Lambda\left[P \dot{\mathbf{a}}\left(\omega_{k}\right)-\frac{\dot{\sigma}\left(\omega_{k}\right)}{\sigma\left(\omega_{k}\right)} P \mathbf{a}\left(\omega_{k}\right)\right]+\mathbf{b}^{\mathrm{T}}\left(\omega_{k}\right) P \Lambda\left[P \dot{\mathbf{b}}\left(\omega_{k}\right)-\frac{\dot{\sigma}\left(\omega_{k}\right)}{\sigma\left(\omega_{k}\right)} P \mathbf{b}\left(\omega_{k}\right)\right]=0,
$$

where $\Lambda$ is the selection matrix as defined above. 
Proof. The extremal condition is given by

$$
\sum_{i=1}^{l} \frac{\partial P_{s_{i} s_{i}}}{\partial \omega_{k}}=0
$$

Using relation (13a) this becomes

$$
\begin{aligned}
& \sum_{i=1}^{l} \sum_{j, s}\left[\left\{P_{s_{i} j} \dot{a}_{j}\left(\omega_{k}\right) a_{s}\left(\omega_{k}\right) P_{s s_{i}}+P_{s_{i} j} \dot{b}_{j}\left(\omega_{k}\right) b_{s}\left(\omega_{k}\right) P_{s s_{i}}\right\}\right. \\
& \left.\quad-\frac{\dot{\sigma}\left(\omega_{k}\right)}{\sigma\left(\omega_{k}\right)}\left\{P_{s_{i} j} a_{j}\left(\omega_{k}\right) a_{s}\left(\omega_{k}\right) P_{s s_{i}}+P_{s_{i} j} b_{j}\left(\omega_{k}\right) b_{s}\left(\omega_{k}\right) P_{s s_{i}}\right\}\right]=0
\end{aligned}
$$

from which the results follows.

Corollary 3. The extremal values of $\operatorname{trace}(P)$ are given by the relation

$$
a^{\mathrm{T}}\left(\omega_{k}\right) P^{2} \dot{a}\left(\omega_{k}\right)+b^{\mathrm{T}}\left(\omega_{k}\right) P^{2} \dot{b}\left(\omega_{k}\right)=0,
$$

when $\dot{\sigma}(\omega)=0$. This corresponds to $R=\sigma_{0} \operatorname{diag}(1,1, \ldots, 1)$.

Proof. For this case $\Lambda=I$ and the result follows.

Corollary 4. If either $f\left(\omega_{k}\right)$ or $g\left(\omega_{k}\right)$ is nonzero, then there exists no $\omega_{k}, \omega_{k}>0$, for which the vectors $P \mathbf{a}\left(\omega_{k}\right)$ and $P \mathbf{b}\left(\omega_{k}\right)$ equal zero, provided $P$ is nonsingular.

Proof. Noting that the vector $\mathrm{Pa}$ is proportional to

$$
\left\{\begin{array}{l}
f\left(\omega_{k}\right)\left[B-\omega_{k}^{2} A\right] \\
g\left(\omega_{k}\right)\left[B^{2}-A C\right] \\
f\left(\omega_{k}\right)\left[C-\omega_{k}^{2} B\right]
\end{array}\right\}
$$

where $A, B$ and $C$ are defined in relation (21), the result follows. The result for $P \mathbf{b}$ is along the same lines.

Corollary 5. If $l>1$, there exists no frequency $\omega_{k}, \omega_{k} \in I_{\Omega}$, such that for $f\left(\omega_{k}\right)$ and $g\left(\omega_{k}\right)$ nonzero and $P$ nonsingular

$$
\Lambda P \mathbf{a}=\mathbf{0} .
$$

Proof. The result follows from Corollary 4 and relations (22).

We note therefore that, in general, the data at each frequency provide information on $m$ and/or $k$.

\section{Numerical example.}

$$
\text { Let } q(t)=\left\{\begin{array}{lr}
0, & t<0 \\
e^{-\beta t}, & t \geqslant 0, \beta>0
\end{array}\right\}
$$

Then, $f(\omega)=\beta$ and $g(\omega)=\omega$. The vectors a and b become

and

$$
\mathbf{a}=\left[-\omega^{2} \beta-\omega^{2} \beta\right]^{\mathrm{T}},
$$

$$
\mathbf{b}=\left[-\omega^{3} \omega \beta \omega\right]^{\mathrm{T}}
$$


Let us assume that measurements at $\omega=\omega_{1}, \omega_{2}, \ldots, \omega_{k-1}$ have been made and the next measurement is to be taken at $\omega_{k}$. Let $P^{-}$denote the covariance matrix at the end of the first $(k-1)$ measurements, and $\omega_{i} \in[1, \infty)$. Let $\sigma\left(\omega_{i}\right)=\sigma_{0}, \forall i$, so that $\dot{\sigma}(\omega) \equiv 0$.

If $\omega_{k}$ is such that $\sum P_{3 j} a_{j}\left(\omega_{k}\right)=0$, then no improvement in the variance of the stiffness estimate can be expected by obtaining the additional measurement at $\omega=\omega_{k}$. This condition for the forcing function (39), after some algebra, implies

$$
\omega_{k}=\left[C^{\prime} / B^{\prime}\right]^{1 / 2}
$$

where

$$
C^{\prime}=\sum_{s=1}^{k-1} \omega_{s}^{4}\left(\beta^{2}+\omega_{s}^{2}\right) \quad \text { and } \quad B^{\prime}=\sum_{s=1}^{k-1} \omega_{s}^{2}\left(\beta^{2}+\omega_{s}^{2}\right) .
$$

For illustration, assume that $k=4$ and $\beta=1$. If the first three measurements are taken at $\omega=1 \mathrm{rad} / \mathrm{sec} ., \omega=2 \mathrm{rad} / \mathrm{sec} ., \omega=3 \mathrm{rad} / \mathrm{sec}$., and $\sigma_{j}=\sigma_{0}, j=1,2,3,4$, then

$$
P^{-}=\sigma_{0}^{2}\left[\begin{array}{ccc}
.00648 & 0 & .04274 \\
0 & .0089 & 0 \\
.04274 & 0 & .3405
\end{array}\right],
$$

$B^{\prime}=112, C^{\prime}=892$, and relation (41) gives $\omega_{4}=2.82 \mathrm{rad} / \mathrm{sec}$. Thus a measurement at $\omega=2.82 \mathrm{rad} / \mathrm{sec}$. will yield no improvement of the covariance of the stiffness. In fact, from Corollary 1 we know that $P_{3 j}^{+}=P_{3 j}^{-}, j=1,2,3$. Again if $\omega_{k}$ is such that $\sum P_{1 j} a_{j}\left(\omega_{k}\right)$ $=0$, then the new measurement will provide no additional information on the mass parameter. After some algebra, we find that this relation gives

$$
\omega_{k}=\left(B^{\prime} / A^{\prime}\right)^{1 / 2} \text {, }
$$

where $A^{\prime}=\sum_{s=1}^{k-1}\left(\beta^{2}+\omega_{s}^{2}\right)$, and $B^{\prime}$ is as defined before. We then have $P_{1 j}^{+}=P_{1 j}^{-}$, $j=1,2,3$. For the example taken, $A^{\prime}=17$, and the value of $\omega_{k}$ satisfying (42) is 2.56 $\mathrm{rad} / \mathrm{sec}$.

For optimally locating the measurement $\omega=\omega_{k}$ so that $P_{11}$ is minimized, equation (34a) needs to be satisfied. This yields

$$
-2 \beta^{2} \sum_{s=1}^{k} \alpha^{2}\left(\omega_{s}\right)+3 \omega_{k}^{2} \sum_{s=1}^{k} \alpha^{2}\left(\omega_{s}\right)+\sum_{s=1}^{k} \omega_{s}^{2} \alpha^{2}\left(\omega_{s}\right)=0
$$

which simplifies to

$$
2 \omega_{k}^{4}+\omega_{k}^{2}\left(4 \beta^{2}+3 A^{\prime}\right)+2 A^{\prime} \beta^{2}-B^{\prime}+2 \beta^{2}=0 .
$$

For $\omega_{4} \in[1, \infty)$ in our example, relation (43) gives a value of 1.148 rads $/ \mathrm{sec}$.

Extensions to some nonlinear sdof-systems. In this section we extend the results obtained dealing with the parameter identification of a linear system to nonlinear systems that can be described by one degree of freedom. The proofs of all the results follow suit from those of the previous section and therefore have been omitted. Here we shall work directly in the time domain.

Consider a structure modelled by a nonlinear differential equation

$$
m \ddot{x}+f(x, \dot{x})=q(t), \quad x(0)=\dot{x}(0)=0,
$$


where within a certain range of response the nonlinear term can be approximated by

$$
f(x, \dot{x})=\sum_{n, m} \beta_{n m} x^{t}(t) \dot{x}^{m}(t) .
$$

The system is assumed to be nonhysteretic. Let us say that the response of the system is measured at times $t=t_{k}, k=1,2, \ldots, t \in(0, T)$, and that the aim is to locate the instants $t=t_{k}$ when measurements should be made so that the data collected thereat would yield the minimal variance of any one of the parameters $m$ or $\beta_{m n}$ whose accurate identification is required. We shall assume that $x, \dot{x}$ and $\ddot{x}$ and their derivatives are continuous functions of time for $t \in(0, T)$.

If the complete time histories $x, \dot{x}$ and $\ddot{x}$ were available (actually they are not), then equation (46) could be rewritten as

$$
m z_{1}(t)+c_{1} z_{2}(t)+c_{2} z_{3}(t)+\cdots+c_{L} z_{L+1}(t)=q(t),
$$

where the $z$ 's correspond to the corresponding time functions, $L=m \times n$ and the coefficients $\beta_{m n}$ are assembled into a one-dimensional array c. Identification of the parameter vector $\boldsymbol{\theta}=\left[\begin{array}{ll}m & \mathbf{c}^{\mathrm{T}}\end{array}\right]^{\mathrm{T}}$ under noisy measurement conditions would lead to the relations

$$
\mathbf{q}=H \theta+\varepsilon,
$$

where

$$
\begin{aligned}
\mathbf{q} & =\left[q\left(t_{1}\right) q\left(t_{2}\right) \because q\left(t_{N}\right)\right]^{\mathrm{T}}, \\
H & =\left[\mathbf{z}\left(t_{1}\right) z\left(t_{2}\right) \cdots \mathbf{z}\left(t_{L+1}\right)\right]^{\mathrm{T}}, \quad \mathbf{z}(t)=\left[z_{1}(t) z_{2}(t) \cdots z_{L+1}(t)\right]^{\mathrm{T}}, \\
\boldsymbol{\varepsilon} & =\text { the zero-mean white measurement noise. }
\end{aligned}
$$

The covariance of the estimate can be written, as before, as the $(1+m n) \times(1+m n)$ matrix

$$
P=\left(H^{\mathrm{T}} R^{-1} H\right)^{-1}
$$

where $R$ is the noise covariance matrix and is taken to be diag $\left(\sigma_{1}^{2}, \sigma_{2}^{2}, \ldots \sigma_{N}^{2}\right)$. Once again the extremal condition for $P$ with respect to a measurement instant $t_{k}$ can be expressed, as before, by the relation

$$
\frac{\boldsymbol{\sigma}_{k}^{2}}{2}\left(\frac{\partial P}{\partial t_{k}}\right)_{r r}=-\left[\sum_{j} P_{r j} z_{j}\left(t_{k}\right)\right]\left[\sum_{s} P_{s r} \dot{z}_{s}\left(t_{k}\right)\right]+\frac{\dot{\boldsymbol{\sigma}}\left(t_{k}\right)}{\sigma\left(t_{k}\right)}\left(\sum_{j} P_{r j} z_{j}\right)^{2}=0,
$$

where $z_{j}$ is the $j$ th element of $\mathbf{z}$. (We note that this result follows directly from equation (14) if we set $\mathbf{b}$ to $0, \mathbf{a}$ to $\mathbf{z}$, and $\omega_{k}$ to $t_{k}$.) We then have

THEOREM 5. For a given forcing function and any $r \in\{1,2, \ldots, m n+1\}$, there may exist times $t_{k}$ such that the inclusion of data at those times does not yield any improvement in the variance $P_{r r}$ of our estimate of the $r$ th parameter. Specifically, when for some $t_{k} \in(0, T)$

$$
\sum_{j=1}^{m n=1} P_{r j} z_{j}\left(t_{k}\right)=0
$$

is satisfied, then $t=t_{k}$ is such an instant.

Proof. The proof follows along the lines of Theorem 1. 
CoRollary 6. For values of $t_{k}$ for which $\sum P_{r j} z_{j}\left(t_{k}\right)=0$, the variance of the estimate of the $r$ th variable as well as its cross-covariance is unaffected by the measurement at $t=t_{k}$.

Proof. As before, the condition implies that

$$
P_{r j}^{+}=P_{r j}^{-}, \quad j=1,2, \ldots m n+1 .
$$

The proof follows, as did that of Corollary 1, from the proof of Theorem 5 .

TheOREM 6. The optimal measurement times $t_{k}, t_{k} \in(0, T)$, if they exist at all, which minimize the variance $P_{r r}$ satisfy the following relation

$$
\sum_{s=1}^{m n+1} P_{s r} \dot{z}_{s}\left(t_{k}\right)=\frac{\dot{\sigma}\left(t_{k}\right)}{\sigma\left(t_{k}\right)} \sum_{j=1}^{m n+1} P_{r j} z_{j} .
$$

Proof. The proof follows along the lines of Theorem 2.

THEOREM 7. The measurement times $t_{k} \in(0, T)$ which cause

$$
\sum_{i=1}^{l} P_{s_{i} s_{i}}, \quad l \leqslant m n+1
$$

to be extremal are given by the relation

$$
\mathbf{z}^{\mathrm{T}}\left(t_{k}\right) P \Lambda P \dot{\mathbf{z}}\left(t_{k}\right)=\frac{\dot{\boldsymbol{\sigma}}\left(t_{k}\right)}{\sigma\left(t_{k}\right)} \mathbf{z}^{\mathrm{T}}\left(t_{k}\right) P \Lambda P \mathbf{z}\left(t_{k}\right),
$$

when $\Lambda$ is the zero matrix whose $n$th diagonal element is 1 if $n=s_{i}, i=1, \ldots, l$, $s_{i} \in(1, \ldots, m n+1)$.

Proof. The proof is exactly along the lines of Theorem 4.

THEOREM 8. If $\mathbf{z}\left(t_{k}\right)$ is such that for a given set $s_{i}, i=1,2, \ldots, l, l \leqslant m n+1$,

$$
\Lambda P \mathbf{z}\left(t_{k}\right)=\mathbf{0} \text {, }
$$

then therre exist times $t_{k} \in(0, T)$ such that $\sum_{i=1}^{l} P_{s_{i} s_{i}}$ remains unaffected. In fact, $P_{r s_{i}}$, $r=1,2, \ldots, m n+1, i=1,2, \ldots, l$ remain unchanged by the new measurement at time $t_{k}$.

Proof. The proof follows along the same lines as that of Theorem 1.

Remarks and conclusions. In this paper we have tried to understand the optimal measurement strategy for identifying the parameters of a single-degree-of-freedom dynamic system.

For a linear system, we have shown that, in general, data acquired at all frequencies, in the inteval $\omega_{k} \in I_{\Omega}$, do not equally enhance our knowledge of the parameters being estimated. Specifically, one can often, given a data stream collected at frquencies $\omega_{i}$, $i=1,2, \ldots, k-1$, forecast the next frequency at which data collection would be maximally beneficial to obtaining a more confident estimate of any one of the desired parameters. Likewise, one can predict the frequency at which data collection would have no influence on improving the uncertainty in our estimate of any desired parameter. It is shown that for $\left\{\omega \in I_{\Omega} \mid f(\omega), g(\omega) \neq 0\right\}$, data at all the frequencies carry information about the damping parameter $c$. Also as opposed to the optimal measurement location $\omega_{k}$ 
for identification of $m$ and $k$, which do depend on the previous measurement locations $\omega_{1}, \omega_{2}, \ldots, \omega_{k-1}$, the optimal locations for the identification of $c$ do not depend on the locations of the measurement stream. They are solely controlled by the nature of the forcing functions used in the identification procedure.

Irrespective of which parameter is being identified, the optimal measurement locations do not depend on the values of the parameters. It is noted that the solution of equations (34) and (35) may not exist for any $\omega$ belonging to the open interval $I_{\Omega}$. For such situations the optimal locations would have to be chosen as the end $\operatorname{point}(s)$ of the interval. Also, it is observed that the optimal measurement locations do not depend on the system output $x(t)$. Thus the optimal locations can be calculated a priori to obtaining the measurement stream. A numerical example has been included to illustrate the analytical results obtained. Furthermore, analytical results relating to minimization of one or some sum of the variances of the estimates are also provided.

The results are extended to nonhysteretic nonlinear oscillators when time data of displacement, velocity and acceleration can be obtained for a given time-dependent forcing function. It is shown that there may exist certain times $t_{k}$ at which measurements provide no additional information regarding any one of the parameters desired to be most accurately estimated. Similarly there exist times $t_{h}$ at which measurements provide maximal information about a desired parameter. The relations that these times $t_{h}$ satisfy in each of the two cases have been analytically deduced.

The results of this paper, it is hoped, will shed light on the manner in which experimentation can be performed so that the amount of data handling and reduction required could perhaps be significantly decreased in the dynamic testing of structural and mechanical systems.

\section{REFERENCES}

[1] D. E. Hudson, Resonance testing of full-scale structures, Proc. ASCE. J. Eng. Mech.. (1964)

[2] F. E. Udwadia and D. K. Sharma. Some uniqueness results in huilding structural identification. SIAM I. Appl. Math. 34, 1 (1978)

[3] W. O. Keightley, Vibrational characteristics of earth dams, Bull. Seismological Soc. Amer.. 56 (1966)

[4] P. C. Shah and F. E. Udwadia, Optimal sensor locations in building structural identification, J. Appl. Mech., 45. (1978)

[5] A. P. Sage and J. L. Melsa, Estimation theory with applications to communications and control. McGraw Hill, 1971 